\title{
Strong coupling from the $\tau$-lepton hadronic width
}

\section{Gauhar Abbas*}

Institute of Mathematical Sciences, Chennai, India

E-mail: gauhareimsc.res.in

\section{B. Ananthanarayan}

Centre for High Energy Physics,

Indian Institute of Science, Bangalore 560 012, India

\section{Irinel Caprini}

Horia Hulubei National Institute for Physics and Nuclear Engineering, P.O.B. MG-6, 077125 Magurele, Romania

\section{Jan Fischer}

Institute of Physics, Academy of Sciences of the Czech Republic,

CZ-182 21 Prague 8, Czech Republic

The strong coupling is determined using a novel renormalization-group-summed perturbation theory and a non power renormalization-group-summed perturbation theory with a tamed large-order behaviour. In this talk, the main features of these two schemes are presented and are applied to the extraction of $\alpha_{s}\left(M_{\tau}^{2}\right)$ from the hadronic decay width of the $\tau$ lepton. While the renormalizationgroup-summed perturbation theory leads to a value of strong coupling close to that from contourimproved perturbation theory, the non power renormalization-group-summed perturbation theory yields a value closer to the standard fixed-order perturbation theory.

The European Physical Society Conference on High Energy Physics

18-24 July, 2013

Stockholm, Sweden

\footnotetext{
*Speaker.
} 


\section{Introduction}

The strong coupling is one of the most important parameters of the Standard Model. Its precise determination at various scales is crucial for testing the asymptotic freedom of QCD, the modern theory of strong interactions. The inclusive hadronic decay width of the $\tau$ lepton provides a clean way to determine $\alpha_{s}$ at low energies [1,2,3,4]. The $R$ ratio for the $\tau$ decays is defined as:

$$
R_{\tau} \equiv \frac{\Gamma\left[\tau^{-} \rightarrow \text { hadrons } v_{\tau}\right]}{\Gamma\left[\tau^{-} \rightarrow e^{-} \bar{v}_{e} v_{\tau}\right]}
$$

Here we are interested in the $\tau$ decay rate into the lightest ( $u$ and $d$ ) quarks, which proceeds either through the vector $(V)$ or axial-vector current $(A)$. This quantity can be expressed theoretically in the form

$$
R_{\tau, V / A}=\frac{N_{c}}{2} S_{\mathrm{EW}}\left|V_{u d}\right|^{2}\left[1+\delta^{(0)}+\delta_{\mathrm{EW}}^{\prime}+\sum_{D \geq 2} \delta_{u d}^{(D)}\right],
$$

where $S_{\mathrm{EW}}=1.0198 \pm 0.0006$ and $\delta_{\mathrm{EW}}^{\prime}=0.0010 \pm 0.0010$ are electroweak corrections, and $\delta_{u d}^{(D)}$ is the contribution from the higher $D$-dimensional operators which arise in the operator product expansion. Our main interest is in the perturbative correction $\delta^{(0)}$ which can be written as [5]

$$
\delta^{(0)}=\frac{1}{2 \pi i} \oint_{|s|=M_{\tau}^{2}} \frac{d s}{s}\left(1-\frac{s}{M_{\tau}^{2}}\right)^{3}\left(1+\frac{s}{M_{\tau}^{2}}\right) \hat{D}_{\text {pert }}(a, L),
$$

where $a \equiv a\left(\mu^{2}\right) \equiv \alpha_{s}\left(\mu^{2}\right) / \pi, L \equiv \ln \frac{-s}{\mu^{2}}$ and $\hat{D}_{\text {pert }}(a, L)$, is the perturbation expansion of the Adler function. In the 'fixed-order perturbation theory' (FOPT) this expansion has the form [6]

$$
\hat{D}_{\mathrm{FOPT}}(a, L)=\sum_{n=1}^{\infty} a^{n} \sum_{k=1}^{n} k c_{n, k} L^{k-1}
$$

while in renormalization-group-improved or 'contour-improved perturbation theory' (CIPT) the series is written as $[7,8]$

$$
\hat{D}_{\mathrm{CIPT}}\left(\alpha_{s}(-s) / \pi, 0\right)=\sum_{n=1}^{\infty} c_{n, 1}\left(\frac{\alpha_{s}(-s)}{\pi}\right)^{n}
$$

In the above expansions the coefficients $c_{n, 1}$ are known for $n \leq 4$ from perturbative calculations in the $\overline{\mathrm{MS}}$-renormalization scheme (see [9] and references therein). The numerical values for $n_{f}=3$ flavours are:

$$
c_{1,1}=1, \quad c_{2,1}=1.640, \quad c_{3,1}=6.371, \quad c_{4,1}=49.076 .
$$

For the next coefficient $c_{5,1}$ estimates are given in $[6,10,11,12]$.

The coefficients $c_{n, j}$ for $j>1$ are determined in terms of $c_{n, 1}$ and the coefficients $\beta_{j}$ of the $\beta$-function of the renormalization group equation (RGE). At present this function is calculated to four loops in the $\overline{\mathrm{MS}}$-renormalization scheme (see $[13,14]$ and references therein), where the first coefficients are:

$$
\beta_{0}=9 / 4, \quad \beta_{1}=4, \quad \beta_{2}=10.0599, \quad \beta_{3}=47.228
$$




\section{Renormalization-Group-Summed Perturbation Theory}

We use a method based on the explicit summation of all renormalization group accessible logarithms $[15,16,17]$, which was recently applied to the hadronic width of the $\tau$ lepton $[18,19$, 20, 21]. In this method, the Adler function is written as

$$
\hat{D}_{\mathrm{RGSPT}}(a, L)=\sum_{n=1}^{\infty} a^{n} D_{n}(a L),
$$

where

$$
D_{n}(a L) \equiv \sum_{k=n}^{\infty}(k-n+1) c_{k, k-n+1}(a L)^{k-n} .
$$

We now exploit the fact that the Adler function defined by the expansion (1.4) is scale independent

$$
\mu^{2} \frac{\mathrm{d}}{\mathrm{d} \mu^{2}}\left[\hat{D}_{\mathrm{FOPT}}(a, L)\right]=0 \Rightarrow \beta(a) \frac{\partial \hat{D}_{\mathrm{FOPT}}}{\partial a}-\frac{\partial \hat{D}_{\mathrm{FOPT}}}{\partial L}=0 .
$$

The relevant renormalization group equation $(\mathrm{RGE})$ is written as:

$$
\begin{aligned}
0 & =-\sum_{n=1}^{\infty} \sum_{k=2}^{n} k(k-1) c_{n, k} a^{n} L^{k-2} \\
& -\left(\beta_{0} a^{2}+\beta_{1} a^{3}+\beta_{2} a^{4}+\ldots+\beta_{l} a^{l+2}+\ldots\right) \times \sum_{n=1}^{\infty} \sum_{k=1}^{n} n k c_{n, k} a^{n-1} L^{k-1} .
\end{aligned}
$$

By extracting the aggregate coefficient of $a^{n} L^{n-p}$ we obtain the recursion formula $(n \geq p)$

$$
0=(n-p+2) c_{n, n-p+2}+\sum_{\ell=0}^{p-2}(n-\ell-1) \beta_{\ell} c_{n-\ell-1, n-p+1} .
$$

Multiplying both sides of (2.5) by $(n-p+1)(a L)^{n-p}$ and summing from $n=p$ to $\infty$, we obtain a system of first-order linear differential equation for the functions defined in (2.2), written as

$$
\frac{\mathrm{d} D_{n}}{\mathrm{~d}(a L)}+\sum_{\ell=0}^{n-1} \beta_{\ell}\left((a L) \frac{\mathrm{d}}{\mathrm{d}(a L)}+n-\ell\right) D_{n-\ell}=0
$$

for $n \geq 1$, with the initial conditions $D_{n}(0)=c_{n, 1}$ which follow from (2.2). The solution of the above equations can be found iteratively in an analytical closed form. The first two solutions are

$$
D_{1}(a L)=\frac{c_{1,1}}{y}, \quad D_{2}(a L)=\frac{c_{2,1}}{y^{2}}-\frac{\beta_{1} c_{1,1} \ln y}{\beta_{0} y^{2}}, \quad y=1+\beta_{0} a L
$$

By inserting in the integral (1.3) the RGSPT expansion (2.1) of the Adler function we obtain the expansion of $\delta^{(0)}$

$$
\delta_{\mathrm{RGSPT}}^{(0)}=\sum_{n=1}^{\infty} a\left(M_{\tau}^{2}\right)^{n} d_{n}, \text { where } d_{n}=\frac{1}{2 \pi i} \oint_{|s|=M_{\tau}^{2}} \frac{d s}{s}\left(1-\frac{s}{M_{\tau}^{2}}\right)^{3}\left(1+\frac{s}{M_{\tau}^{2}}\right) D_{n}(a L) .
$$

In Table 1 we show the behaviour of the FOPT, CIPT and RGSPT as functions of the truncation order $N$ of the series. For $N=4$, the difference between the results of the RGSPT and the standard FOPT is 0.01754 , and the difference from the RGSPT and CIPT is 0.0039 , which confirms that the RGSPT gives results close to those of the CIPT. 


\begin{tabular}{llcc}
\hline & $\delta_{\text {FOPT }}^{(0)}$ & $\delta_{\text {CIPT }}^{(0)}$ & $\delta_{\text {RGSPT }}^{(0)}$ \\
\hline$N=1$ & 0.1082 & 0.1479 & 0.1455 \\
$N=2$ & 0.1691 & 0.1776 & 0.1797 \\
$N=3$ & 0.2025 & 0.1898 & 0.1931 \\
$N=4$ & 0.2199 & 0.1984 & 0.2024 \\
$N=5$ & 0.2287 & 0.2022 & 0.2056 \\
\hline
\end{tabular}

Table 1: Predictions of $\delta^{(0)}$ in the standard FOPT, CIPT and the RGSPT, for various truncation orders $N$ using $\alpha_{s}\left(M_{\tau}^{2}\right)=0.34$.

\section{Determination of $\alpha_{s}$ from RGSPT expansion}

We adopt as input the recent phenomenological value [6, 12]

$$
\delta_{\text {phen }}^{(0)}=0.2037 \pm 0.0040_{\exp } \pm 0.0037_{\mathrm{PC}} .
$$

Using the expansion (2.8) truncated at $N=5$, with the known coefficients $c_{n, 1}$ from (1.6) and the conservative choice $c_{5,1}=283 \pm 283$ as in [6], we obtain [18]

$$
\left.\alpha_{s}\left(M_{\tau}^{2}\right)=0.3378 \pm 0.0046_{\mathrm{exp}} \pm 0.0042_{\mathrm{PC}}^{+0.0062}+c_{5,1}\right)_{-0.0004}^{+0.0005}(\mathrm{scale}){ }_{-0.000082}^{+0.000085}\left(\beta_{4}\right),
$$

where the errors from various sources are indicated separately (in the last term we used the estimate $\beta_{4}= \pm \beta_{3}^{2} / \beta_{2}$ for next coefficient in the expansion of the $\beta$ function). Combining the errors in quadrature

$$
\alpha_{s}\left(M_{\tau}^{2}\right)=0.338 \pm 0.010
$$

\section{RGS Non-Power Perturbation Theory}

One of the ambiguites in the extraction of $\alpha_{s}$ from the hadronic $\tau$ decays arises from the large-order behaviour of the QCD perturbative series. The large-order behaviour of the RGSPT expansion was investigated in [19] in a model of the Adler function proposed in [6]. In this model, the RGS expansion of the QCD Adler function has a behaviour which is similar to that of CIPT and eventually exhibits large oscillations, thereby showing the divergent character of the QCD perturbative series. We improve this behaviour of the RGSPT expansion by the analytical continuation in the Borel plane. The method was applied to FOPT and CIPT by Caprini and Fischer [22, 23, 24].

The large-order behaviour of the perturbation theory is encoded in the singularities of the Borel transform $B(u)$, defined starting from the expansion (1.5) as

$$
B(u)=\sum_{n=0}^{\infty} c_{n+1,1} \frac{u^{n}}{\beta_{0}^{n} n !} .
$$

The function $B(u)$ has singularities placed on the real axis along the lines $u \leq-1$ and $u \geq 2$. Therefore, the Taylor expansion (4.1) converges only in the disk $|u|<1$, limited by the nearest singularity at $u=-1$ of the expanded function. The region of convergence can be enlarged if 
the series in powers of $u$ is replaced by a series in powers of an "optimal" variable $\widetilde{w}(u)$ that conformally maps the holomorphy domain of $B(u)$, i.e. the $u$-plane with cut along $u \geq 2$ and $u \leq$ -1 , onto the unit disk $|w|<1$ of the complex plane $w \equiv \widetilde{w}(u)$. This also accelerates the convergence rate at all points in the holomorphy domain [24, 25].

The Borel transform of the RGSPT expansion (2.1) is written as [19]

$$
B_{\mathrm{RGSPT}}(u, y)=B(u)+\sum_{n=0}^{\infty} \frac{u^{n}}{\beta_{0}^{n} n !} \sum_{j=1}^{n} c_{j, 1} d_{n+1, j}(y),
$$

where $y=1+\beta_{0} a L$. We consider a general class of conformal mappings

$$
\widetilde{w}_{l m}(u)=\frac{\sqrt{1+u / l}-\sqrt{1-u / m}}{\sqrt{1+u / l}+\sqrt{1-u / m}}, \quad l \geq 1, m \geq 2
$$

where $l, m$ are positive integers satisfying $l \geq 1$ and $m \geq 2$. The function $\widetilde{w}_{l m}(u)$ maps the $u$-plane cut along $u \leq-l$ and $u \geq m$ onto the disk $\left|w_{l m}\right|<1$ in the plane $w_{l m} \equiv \widetilde{w}_{l m}(u)$. We define further the class of compensating factors of the simple form

$$
S_{l m}(u)=\left(1-\frac{\widetilde{w}_{l m}(u)}{\widetilde{w}_{l m}(-1)}\right)^{\gamma_{1}^{(l)}}\left(1-\frac{\widetilde{w}_{l m}(u)}{\widetilde{w}_{l m}(2)}\right)^{\gamma_{2}^{(m)}}
$$

where the exponents

$$
\begin{gathered}
\gamma_{1}^{(l)}=\gamma_{1}\left(1+\delta_{l 1}\right), \quad \gamma_{2}^{(m)}=\gamma_{2}\left(1+\delta_{m 2}\right), \\
\gamma_{1}=1.21, \quad \gamma_{2}=2.58,
\end{gathered}
$$

are chosen such that $S_{l m}(u)$ cancels the dominant singularities of $B_{\mathrm{RGSPT}}(u, y)$, situated at $u=-1$ and $u=2$. We further expand the product $S_{l m}(u) B_{\mathrm{RGSPT}}(u, y)$ in powers of the variable $\widetilde{w}_{l m}(u)$, as

$$
S_{l m}(u) B_{\mathrm{RGSPT}}(u, y)=\sum_{n \geq 0} c_{n, \mathrm{RGSPT}}^{(l m)}(y)\left(\widetilde{w}_{l m}(u)\right)^{n} .
$$

We are thus led to the class of RGSNPPT expansions

$$
\widehat{D}_{\operatorname{RGSNPPT}}(s)=\sum_{n \geq 0} c_{n, \mathrm{RGSPT}}^{(l m)}(y) \mathscr{W}_{n, \mathrm{RGSPT}}^{(l m)}(s),
$$

where

$$
\mathscr{W}_{n, \operatorname{RGSPT}}^{(l m)}(s)=\frac{1}{\beta_{0}} \mathrm{PV} \int_{0}^{\infty} \exp \left(\frac{-u}{\beta_{0} \tilde{a}_{s}(-s)}\right) \frac{\left(\widetilde{w}_{l m}(u)\right)^{n}}{S_{l m}(u)} \mathrm{d} u .
$$

The coefficients $c_{n, \mathrm{RGSPT}}^{(l m)}(y)$ are defined by the expansion (4.6) and the coupling $\widetilde{a}_{s}(-s)$ appearing in the Laplace-Borel integral is the one-loop solution of the RGE.

\section{High-order behaviour of RGSNPPT expansions}

In Table 2 we record the remarkable supression of the divergent behaviour of the RGSPT expansion through analytic continuation in the Borel plane. 


\begin{tabular}{cccccccc}
\hline$N$ & CIPT & FOPT & RGSPT & RGSNPPT $w_{12}$ & RGSNPPT $w_{13}$ & RGSNPPT $w_{1 \infty}$ & RGSNPPT $w_{23}$ \\
\hline 2 & -0.0595 & -0.0679 & -0.0574 & -0.0347 & -0.0239 & -0.0417 & -0.0177 \\
3 & -0.0473 & -0.0345 & -0.0440 & -0.0333 & -0.0301 & -0.0349 & -0.0303 \\
4 & -0.0388 & -0.0171 & -0.0347 & -0.0089 & -0.0142 & -0.0067 & -0.0132 \\
5 & -0.0349 & -0.0083 & -0.0315 & -0.0070 & -0.0086 & -0.0058 & -0.0070 \\
6 & -0.0325 & -0.0043 & -0.0284 & -0.0073 & -0.0071 & -0.0064 & -0.0072 \\
7 & -0.0325 & -0.0029 & -0.0298 & -0.0059 & -0.0057 & -0.0056 & -0.0044 \\
8 & -0.0354 & -0.0018 & -0.0309 & -0.0041 & -0.0035 & -0.0041 & -0.0011 \\
9 & -0.0367 & -0.0004 & -0.0363 & -0.0023 & -0.0019 & -0.0028 & -0.0010 \\
10 & -0.0529 & 0.0019 & -0.0483 & 0.0014 & -0.0012 & -0.0020 & 0.0004 \\
11 & -0.0409 & 0.0031 & -0.0458 & 0.0036 & -0.0008 & -0.0016 & -0.0009 \\
12 & -0.1248 & 0.0065 & -0.1335 & 0.0031 & -0.0006 & -0.0015 & 0.0005 \\
13 & 0.0258 & 0.0037 & 0.0534 & 0.0026 & -0.0004 & -0.0015 & -0.0005 \\
14 & -0.5286 & 0.0204 & -0.7850 & 0.0018 & -0.0003 & -0.0015 & -0.0011 \\
15 & 0.8640 & -0.0201 & 1.7734 & 0.0006 & -0.0002 & -0.0015 & 0.0044 \\
16 & -3.5991 & 0.1447 & -7.7043 & 0.0001 & $-7 \cdot 10^{-6}$ & -0.0015 & -0.0131 \\
17 & 9.3560 & -0.4252 & 24.8586 & -0.0004 & $4 \cdot 10^{-6}$ & -0.0014 & 0.0238 \\
18 & -31.76 & 1.907 & -94.26 & -0.0013 & -0.0001 & -0.0013 & -0.0310 \\
\hline \hline
\end{tabular}

Table 2: The difference $\delta^{(0)}-\delta_{\text {exact }}^{(0)}$ for the model proposed in [6] for $\alpha_{S}\left(M_{\tau}^{2}\right)=0.34$ with the standard CIPT, FOPT and RGSPT expansions, and the new RGSNPPT expansions for various conformal mappings $w_{l m}$, truncated at order $N$. The exact value $\delta_{\text {exact }}^{(0)}=0.2371$.

\section{Determination of $\alpha_{S}$ from RGSNPPT expansions}

Using the phenomenological input (3.1) and the RGSNPPT expansions (4.7) we obtain [19]

$$
\left.\alpha_{s}\left(M_{\tau}^{2}\right)=0.3189 \pm 0.0034_{\exp } \pm 0.0031_{\mathrm{PC}}^{+0.0105}+\mathrm{c}_{5,1}\right) \pm 0.0010_{\beta_{4}}
$$

and after combining the errors in quadrature

$$
\alpha_{s}\left(M_{\tau}^{2}\right)=0.3189_{-0.0115}^{+0.0145}
$$

By evolving to the scale of $M_{Z}$ our prediction reads

$$
\alpha_{s}\left(M_{Z}^{2}\right)=0.1184_{-0.0015}^{+0.0018} .
$$

\section{Conclusion}

This work is motivated by the well-known discrepancy between the predictions of $\alpha_{s}\left(M_{\tau}^{2}\right)$ from the standard FOPT and CIPT expansions. We have shown that the summation of all the logarithms accesible by renormalization group invariance provides a systematic expansion of the Adler function with a good behaviour in the complex energy plane [18]. The results of the new RGSPT expansion are similar to those obtained with CIPT. We further tamed the divergent character of the perturbative series by the method of conformal mappings of the Borel plane, defining the RGS non-power expansions [19], similar to the FONPPT and CINPPT defined in [22, 24]. The RGSNPPT expansions lead to a prediction for $\alpha_{s}\left(M_{\tau}^{2}\right)$ similar to that obtained with standard FOPT and with CINPPT. As shown recently [26], the good large-order properties of the renormalizationgroup improved non-power expansions are valid also for a large class of moments of the spectral functions (the one associated with the hadronic width being a special one). Therefore, CINPPT and RGSNPPT provide a solid theoretical framework in moment analyses for the simultaneous determination of the strong coupling and other parameters of QCD from hadronic $\tau$ decays. 


\section{References}

[1] J. Beringer et al. (Particle Data Group), Phys. Rev. D86, 010001 (2012).

[2] A. Pich, PoS ConfinementX (2012) 022, arXiv:1303.2262 [hep-ph].

[3] M. Jamin, PoS ConfinementX (2012) 098, arXiv:1302.2425 [hep-ph].

[4] G. Altarelli, PoS Corfu2012 (2013) 002, arXiv:1303.6065 [hep-ph].

[5] E. Braaten, S. Narison and A. Pich, Nucl. Phys. B 373, 581 (1992).

[6] M. Beneke and M. Jamin, JHEP 09, 044 (2008), arXiv:0806.3156 [hep-ph].

[7] A.A.Pivovarov, Z. Phys. C 53, 461 (1992), [Sov. J. Nucl. Phys. 54, 676 (1991)], hep-ph/0302003.

[8] F. Le Diberder and A. Pich, Phys. Lett. B 289, 165 (1992).

[9] P.A. Baikov, K.G. Chetyrkin and J.H. Kühn, Phys. Rev. Lett. 101, 012002 (2008), arXiv:0801.1821 [hep-ph].

[10] M. Davier, S. Descotes-Genon, A. H ocker, B. Malaescu and Z. Zhang, Eur. Phys. J. C56, 305 (2008), arXiv:0803.0979 [hep-ph].

[11] A. Pich, Tau decay determination of the QCD coupling, in Workshop on Precision Measurements of $\alpha_{s}$, ed. S. Bethke et al, page 21, arXiv:1110.0016 [hep-ph].

[12] M. Beneke and M. Jamin, Fixed-order analysis of the hadronic $\tau$ decay width, in Workshop on Precision Measurements of $\alpha_{s}$, ed. S. Bethke et al, page 25, arXiv:1110.0016 [hep-ph].

[13] S.A. Larin, T. van Ritbergen and J.A.M. Vermaseren, Phys. Lett. B400, 379 (1997), hep-ph/9701390; S.A. Larin, T. van Ritbergen and J.A.M. Vermaseren, Phys. Lett. B404, 153 (1997), hep-ph/9702435.

[14] M. Czakon, Nucl. Phys. B710, 485 (2005), hep-ph/0411261.

[15] C.J. Maxwell and A. Mirjalili, Nucl. Phys. B 577, 209 (2000), hep-ph/0002204; Nucl. Phys. B 611, 423 (2001), hep-ph/0103164.

[16] M.R. Ahmady, F. A. Chishtie, V. Elias, A. H. Fariboz, N. Fattahi, D. G. C. McKoen, T. N. Sherry, and T.G. Steele, Phys. Rev. D 66, 014010 (2002), hep-ph/0203183.

[17] M.R. Ahmady, , F. A. Chishtie, V. Elias, A. H. Fariboz, D. G. C. McKoen, T. N. Sherry, A. Sqires, and T.G. Steele, Phys. Rev. D 67, 034017 (2003), hep-ph/0208025.

[18] G. Abbas, B. Ananthanarayan and I. Caprini, Phys. Rev. D 85, 094018 (2012), arXiv:1202.2672 [hep-ph].

[19] G. Abbas, B. Ananthanarayan, I. Caprini and J. Fischer, Phys. Rev. D 87, 014008 (2013), arXiv:1211.4316 [hep-ph].

[20] I. Caprini, Mod. Phys. Lett. A 28, 1360003 (2013), arXiv:1306.0985 [hep-ph].

[21] G. Abbas, B. Ananthanarayan and I. Caprini, Mod. Phys. Lett. A 28, 1360004 (2013), arXiv:1306.1095 [hep-ph].

[22] I. Caprini and J. Fischer, Eur. Phys. J. C64, 35 (2009), arXiv:0906.5211 [hep-ph].

[23] I. Caprini and J. Fischer, Nucl. Phys. B Proc. Suppl., 218, 128 (2011), arXiv:1011.6480 [hep-ph].

[24] I. Caprini and J. Fischer, Phys. Rev. D 84, 054019 (2011), arXiv:1106.5336 [hep-ph].

[25] S. Ciulli and J. Fischer, Nucl. Phys. 24, 465 (1961).

[26] G. Abbas, B. Ananthanarayan, I. Caprini and J. Fischer, Phys. Rev. D 88, 034026 (2013), arXiv:1307.6323 [hep-ph]. 